\title{
Good Linear Operators and Meromorphic Solutions of Functional Equations
}

\author{
Nan $\mathrm{Li}^{1,2}{ }^{1,2}$ Risto Korhonen, ${ }^{2}$ and Lianzhong Yang ${ }^{1}$ \\ ${ }^{1}$ School of Mathematics, Shandong University, Jinan, Shandong 250100, China \\ ${ }^{2}$ Department of Physics and Mathematics, Joensuu Campus, University of Eastern Finland, P.O. Box 111, 80101 Joensuu, Finland \\ Correspondence should be addressed to Risto Korhonen; risto.korhonen@uef.fi
}

Received 23 March 2015; Accepted 24 April 2015

Academic Editor: Bao Qin Li

Copyright (c) $2015 \mathrm{Nan}$ Li et al. This is an open access article distributed under the Creative Commons Attribution License, which permits unrestricted use, distribution, and reproduction in any medium, provided the original work is properly cited.

\begin{abstract}
Nevanlinna theory provides us with many tools applicable to the study of value distribution of meromorphic solutions of differential equations. Analogues of some of these tools have been recently developed for difference, $q$-difference, and ultradiscrete equations. In many cases, the methodologies used in the study of meromorphic solutions of differential, difference, and $q$-difference equations are largely similar. The purpose of this paper is to collect some of these tools in a common toolbox for the study of general classes of functional equations by introducing notion of a good linear operator, which satisfies certain regularity conditions in terms of value distribution theory. As an example case, we apply our methods to study the growth of meromorphic solutions of the functional equation $M(z, f)+P(z, f)=h(z)$, where $M(z, f)$ is a linear polynomial in $f$ and $L(f)$, where $L$ is good linear operator, $P(z, f)$ is a polynomial in $f$ with degree $\operatorname{deg} P \geq 2$, both with small meromorphic coefficients, and $h(z)$ is a meromorphic function.
\end{abstract}

\section{Introduction}

Lemma on the logarithmic derivatives is an important technical tool in the study of value distribution of meromorphic solutions of differential equations. It is one of the key components in the proof of the Clunie lemma [1] and in a theorem due to A. Z. Mohon'ko and V. D. Mohon'ko [2], both of which are applicable to large classes of differential equations. Similarly, the difference analogues of the lemma on the logarithmic derivatives due to Halburd and the second author $[3,4]$ and Chiang and Feng $[5,6]$ are applicable to study large classes of difference equations, often by using methods similar to the case of differential equations. A $q$-difference analogue [7] of the lemma on the logarithmic derivatives, as well as an analogous result on the proximity function of polynomial compositions of meromorphic functions [8], is applicable to corresponding classes of $q$-difference equations and functional equations much in the same way. Therefore it is natural to present all these results under one general framework. For value distribution of meromorphic functions, this was done in [9], where a second main theorem was given for general linear operators, operating on a subfield of meromorphic functions for which a suitable analogue of the lemma on the logarithmic derivative exists. The purpose of this paper is to develop this method further so that it is applicable to equations and to apply it to study meromorphic solutions of a general class of functional equations. This will be done in Section 2 by introducing the notion of a good linear operator, which encompasses such operators as $L(f)=f^{\prime}$, $L_{q}(f)=f(q z)$, and $E_{c}(f)=f(z+c)$. In Section 3 we apply our methods to study the existence and uniqueness and the growth of meromorphic solutions of a general class of functional equations. Sections 4-7 contain the proofs of the results stated in Section 3.

\section{Good Linear Operators}

The lemma on the logarithmic derivative and its difference analogues all produce different types of exceptional sets. In order to include this phenomenon in our setup, we introduce the following notion. We say $\mathbb{P}$ is an exceptional set property if for any two sets $E_{1} \subset(0, \infty)$ and $E_{2} \subset(0, \infty)$ having the property $\mathbb{P}$ it follows that $E_{1} \cup E_{2}$ also has $\mathbb{P}$. For instance, "finite linear measure," "finite logarithmic measure," and "zero logarithmic density" are exceptional set properties. Denote by $\mathscr{M}$ the field of meromorphic functions in the complex plane, 
and let $\mathscr{N} \subset \mathscr{M}$. We say that a linear operator $L: \mathcal{N} \rightarrow \mathscr{N}$ is a good linear operator for $\mathcal{N}$ with exceptional set property $\mathbb{P}$ if the following two properties hold:

(1) For any $f \in \mathscr{N}$,

$$
m\left(r, \frac{L(f)}{f}\right)=o(T(r, f))
$$

as $r \rightarrow \infty$ outside of an exceptional set $E_{f}$ with the property $\mathbb{P}$.

(2) The counting functions $N(r, f)$ and $N(r, L(f))$ are asymptotically equivalent; that is, there is a constant $K \geq 1$ such that

$$
\begin{aligned}
& \frac{1}{K} N(r, f)+o(T(r, f)) \\
& \quad \leq N(r, L(f)) \leq K N(r, f)+o(T(r, f))
\end{aligned}
$$

as $r \rightarrow \infty$ outside of an exceptional set $E_{f}$ with the property $\mathbb{P}$.

For example, if $\mathcal{N}=\mathscr{M}$ and $L(f)=f^{\prime}$, then property (1) is satisfied by the lemma on the logarithmic derivatives with $\mathbb{P}$ being "finite linear measure." Property (2) holds with $K=2$, even without an error term or an exceptional set. Another example is given by taking $\mathcal{N}$ to be the set of all meromorphic functions of hyperorder strictly less than one, and $L(f(z))=$ $f(z+1)$. Then property (1) is satisfied by the difference analogue of the lemma on the logarithmic derivatives with $\mathbb{P}$ being "finite logarithmic measure." In this case, property (2) holds with $K=1$.

The following result shows that a composition of two good operators is also a good operator. Note, however, that the sum of two good linear operators is not necessarily a good operator, since the lower bound in (2) may fail to be valid.

Lemma 1. If $L_{1}$ and $L_{2}$ are good linear operators for $\mathcal{N}$ with exceptional set property $\mathbb{P}$, then $L_{1} \circ L_{2}$ is a good linear operator for $\mathcal{N}$ with the same exceptional set property $\mathbb{P}$.

Proof. Since the linearity follows immediately by the linearity of $L_{1}$ and $L_{2}$, we only need to check that properties (1) and (2) hold for $L_{1} \circ L_{2}$.

First, for any $f \in \mathcal{N}$, we have

$$
\begin{aligned}
m\left(r, \frac{L_{1}\left(L_{2}(f)\right)}{f}\right) \leq & m\left(r, \frac{L_{1}\left(L_{2}(f)\right)}{L_{2}(f)}\right) \\
& +m\left(r, \frac{L_{2}(f)}{f}\right) .
\end{aligned}
$$

Therefore, since $f \in \mathcal{N}$ and $L_{2}(f) \in \mathcal{N}$ and by the assumption that $L_{1}$ and $L_{2}$ are good operators, we have

$$
m\left(r, \frac{L_{1}\left(L_{2}(f)\right)}{f}\right)=o\left(T\left(r, L_{2}(f)\right)\right)+o(T(r, f))
$$

as $r \rightarrow \infty$ outside of a set with exceptional set property $\mathbb{P}$. But since

$$
\begin{aligned}
T\left(r, L_{2}(f)\right)= & m\left(r, L_{2}(f)\right)+N\left(r, L_{2}(f)\right) \\
\leq & m(r, f)+m\left(r, \frac{L_{2}(f)}{f}\right)+K N(r, f) \\
& +o(T(r, f)) \\
\leq & K T(r, f)+o(T(r, f))
\end{aligned}
$$

as $r \rightarrow \infty$ outside of a set with exceptional set property $\mathbb{P}$, (4) becomes

$$
m\left(r, \frac{L_{1}\left(L_{2}(f)\right)}{f}\right)=o(T(r, f)) .
$$

Thus property (1) holds for the operator $L_{1} \circ L_{2}$.

To show that property (2) also holds, we observe that since $L_{2}(f) \in \mathcal{N}, f \in \mathcal{N}$,

$$
\begin{aligned}
& \frac{1}{K_{1}} N\left(r, L_{2}(f)\right)+o\left(T\left(r, L_{2}(f)\right)\right) \\
& \quad \leq N\left(r, L_{1}\left(L_{2}(f)\right)\right) \\
& \quad \leq K_{1} N\left(r, L_{2}(f)\right)+o\left(T\left(r, L_{2}(f)\right)\right), \\
& \frac{1}{K_{2}} N(r, f)+o(T(r, f)) \\
& \quad \leq N\left(r, L_{2}(f)\right) \leq K_{2} N(r, f)+o(T(r, f))
\end{aligned}
$$

as $r \rightarrow \infty$ outside of a set with exceptional set property $\mathbb{P}$, it follows by (5) that

$$
\begin{aligned}
& \frac{1}{K_{1} K_{2}} N(r, f)+o(T(r, f)) \\
& \quad \leq N\left(r, L_{1}\left(L_{2}(f)\right)\right) \\
& \quad \leq K_{1} K_{2} N(r, f)+o(T(r, f)) .
\end{aligned}
$$

Thus property (2) is valid for $L_{1} \circ L_{2}$, and hence it is a good linear operator for $\mathcal{N}$ with exceptional set property $\mathbb{P}$.

As we mentioned in the introduction, the operation of differentiation $L: \mathscr{M} \rightarrow \mathscr{M}, L(f)=f^{\prime}$, is a good linear operator with the exceptional set property "finite linear measure." Lemma 1 implies that a composition of single term differential and difference operators of arbitrary order is a good linear operator for sufficiently slowly growing meromorphic functions.

Lemma 2. Let $c \in \mathbb{C}$ and $k \in \mathbb{N} \cup\{0\}$, and let $\mathcal{N}_{1}$ be the field of meromorphic functions of hyperorder strictly less than one. The operator

$$
L(f(z))=f^{(k)}(z+c)
$$

is a good linear operator in $\mathcal{N}_{1}$ with $\mathbb{P}=$ "finite logarithmic measure." 
In order to prove this lemma, we need the following two results from the field of difference Nevanlinna theory. The first is a difference analogue of the lemma on the logarithmic derivatives.

Lemma 3 (see [10]). Let $f(z)$ be a nonconstant meromorphic function and $c \in \mathbb{C}$. If

$$
\sigma_{2}(f):=\limsup _{r \rightarrow \infty} \frac{\log \log T(r, f)}{\log r}=\sigma_{2}<1
$$

and $\varepsilon>0$, then

$$
m\left(r, \frac{f(z+c)}{f(z)}\right)=o\left(\frac{T(r, f)}{r^{1-\sigma_{2}-\varepsilon}}\right)
$$

for all $r$ outside of a set of finite logarithmic measure.

The second auxiliary lemma helps us to deal with shifted counting functions in the field $\mathcal{N}_{1}$.

Lemma 4 (see [10]). Let $T:[0,+\infty) \rightarrow[0,+\infty)$ be a nondecreasing continuous function and let $s \in(0, \infty)$. If the hyperorder of $T$ is strictly less than one, that is,

$$
\limsup _{r \rightarrow \infty} \frac{\log \log T(r)}{\log r}=\sigma_{2}<1
$$

and $\delta \in\left(0,1-\sigma_{2}\right)$, then

$$
T(r+s)=T(r)+o\left(\frac{T(r)}{r^{\delta}}\right),
$$

where $r$ runs to infinity outside of a set of finite logarithmic measure.

Proof of Lemma 2. By Lemma 1 it is sufficient to show that the operators $L_{1}(f)=f^{\prime}$ and $L_{2}(f(z))=f(z+c)$ are good linear operators in $\mathcal{N}_{1}$ with the exceptional set property $\mathbb{P}$. The operator $L_{1}$ is good in fact in all of $\mathscr{M}$ with a weaker exceptional set property. Namely, property (1) is satisfied by the lemma on the logarithmic derivative, and property (2) holds since

$$
N(r, f) \leq N\left(r, L_{1}(f)\right) \leq 2 N(r, f)
$$

for all meromorphic functions $f \in \mathscr{M}$ and for all $r \geq 1$. By combining (14) with the lemma on the logarithmic derivative, it follows that

$$
T\left(r, L_{1}(f)\right) \leq 2 T(r, f)+o(T(r, f))
$$

as $r \rightarrow \infty$ outside of a set of finite linear measure. Therefore, if $f \in \mathscr{N}_{1}$ it follows that $L_{1}(f) \in \mathcal{N}_{1}$ and thus $L_{1}: \mathcal{N}_{1} \rightarrow$ $\mathcal{N}_{1}$ is a good linear operator in $\mathscr{N}_{1}$ with the exceptional set property $\mathbb{P}$.

If $f \in \mathscr{N}_{1}$, it follows by Lemma 3 that

$$
m\left(r, \frac{L_{2}(f)}{f}\right)=m\left(r, \frac{f(z+c)}{f(z)}\right)=o(T(r, f))
$$

as $r \rightarrow \infty$ outside of a set of finite logarithmic measure. Therefore property (1) is satisfied for $L_{2}$ in $\mathcal{N}_{1}$ with the exceptional set property "finite logarithmic measure." Moreover,

$$
N(r-|c|, f(z)) \leq N\left(r, L_{2}(f)\right) \leq N(r+|c|, f(z))
$$

for all $r \geq|c|$, and so, by Lemma 4, we have

$$
\begin{aligned}
& N(r, f(z))+o(N(r, f)) \\
& \quad \leq N\left(r, L_{2}(f)\right) \leq N(r, f(z))+o(N(r, f))
\end{aligned}
$$

as $r \rightarrow \infty$ outside of a set of finite logarithmic measure. Hence property (2) holds for $L_{2}$ in $\mathscr{N}_{1}$ with the exceptional set property "finite logarithmic measure." Finally, by combining (16) and (18), it follows that

$$
T\left(r, L_{2}(f)\right) \leq T(r, f)+o(T(r, f))
$$

as $r \rightarrow \infty$ outside of a set of finite logarithmic measure. Hence $L_{2}\left(\mathscr{N}_{1}\right) \subset \mathcal{N}_{1}$, and so $L_{2}: \mathcal{N}_{1} \rightarrow \mathcal{N}_{1}$ is a good linear operator in $\mathscr{N}_{1}$ with the exceptional set property $\mathbb{P}$. This completes the proof of Lemma 2.

\section{Meromorphic Solutions of a Functional Equation}

In this section we apply the concept of good linear operator to study meromorphic solutions of

$$
M(z, f)+P(z, f)=h(z),
$$

where $M(z, f)$ denotes a linear polynomial in $f$ and $L(f)$ with $L$ being a good linear operator, $P(z, f)$ is a polynomial in $f$, and $h(z)$ is a meromorphic function.

Equation (20) is an extension of a differential equation studied by Heittokangas et al. [11] in 2002. They considered the growth of meromorphic solutions of

$$
L(f)+P(z, f)=h(z),
$$

where $L(f)=a_{0}(z) f+a_{1}(z) f^{\prime}+\cdots+a_{k}(z) f^{(k)}$ is a linear differential polynomial in $f$ with meromorphic coefficients, $P(z, f)=b_{2}(z) f^{2}+\cdots+b_{n}(z) f^{n}$ is a polynomial in $f$ with meromorphic coefficients, and $h(z)$ is meromorphic, and obtained the following result.

Theorem A. Given $L(f), P(z, f)$, and $h(z)$ as above and $P(z, f) \neq \equiv$, denote by $\mathscr{F}$ the family of meromorphic solutions of (21) such that whenever $f \in \mathscr{F}$, all coefficients of (21) are small meromorphic functions of $f$, and $N(r, f)=S(r, f)$. If now $f, g \in \mathscr{F}$, then

$$
T(r, g)=O(T(r, f))+S(r, f) .
$$

Moreover, if $\alpha>1$, then, for some $r_{\alpha}>0$,

$$
T(r, g)=O(T(\alpha r, f))
$$

for all $r \geq r_{\alpha}$. 
Specialising to $L(f)-p(z) f^{3}=h(z)$, where $p(z)$ is a small meromorphic function, Heittokangas et al. [11] also considered the existence and uniqueness of meromorphic solutions with few poles only and obtained the following result.

Theorem B. Let $f$ be a transcendental meromorphic function. If $f$ satisfies the nonlinear differential equation

$$
a_{1}(z) f^{\prime}+a_{0}(z) f-p(z) f^{3}=h(z),
$$

then one of the following situations hold:

(a) Equation (24) has $f$ as its unique transcendental meromorphic solution such that $N(r, f)=S(r, f)$.

(b) Equation (24) has exactly three transcendental meromorphic solutions $f_{j}, j=1,2,3$, such that $N\left(r, f_{j}\right)=$ $S\left(r, f_{j}\right)$ for $j=1,2,3$. Moreover $a_{1}(z) f_{j}^{\prime}+a_{0}(z) f_{j} \equiv 0$, and $h(z)=-p(z) f_{j}^{3}$ for all $j=1,2,3$.

A differential-difference counterpart of Theorems A and $B$ was obtained by Yang and Laine in [12]. They showed that if $n \geq 4, M(z, f) \not \equiv 0$ is a differential-difference polynomial of $f$, and $h$ is a meromorphic function of finite order, then the equation

$$
f(z)^{n}+M(z, f)=h(z)
$$

possesses at most one admissible transcendental entire solution of finite order and that if such a solution exists, it is of the same order as $h$. Further results on difference and differentialdifference related to (25) can be found, for example, in [13-15].

In the following theorem we apply the concept of good linear operator introduced in Section 2 to obtain a natural extension of Theorem $\mathrm{A}$ and of its difference analogue to a general class of functional equations. In order to state our generalization, we say that meromorphic function $g$ is small with respect to $f$ if $T(r, g)=o(T(r, f))$ as $r \rightarrow \infty$ outside of an exceptional set with the exceptional set property $\mathbb{P}$.

Theorem 5. Let $\mathcal{N} \subset \mathscr{M}$ such that, for any $f \in \mathcal{N}$,

$$
N(r, f)=o(T(r, f))
$$

as $r \rightarrow \infty$ outside of set $E$ with exceptional property $\mathbb{P}$, and let $\left\{L_{k}: k \in J\right\}$ be a finite collection of good linear operators for $\mathcal{N}$ with exceptional set property $\mathbb{P}$. If $f_{1} \in \mathcal{N}$ and $f_{2} \in \mathcal{N}$ are any two meromorphic solutions of the equation

$$
M(z, f)+P(z, f)=h(z)
$$

where $P(z, f)=b_{2}(z) f^{2}+\cdots+b_{n}(z) f^{n}$ is a polynomial in $f$ with small meromorphic coefficients, $h \in \mathcal{M}$, and $M(z, f)$ is a linear polynomial in $f$ and $L_{k}(f), k \in J$, with small meromorphic coefficients, then

$$
T\left(r, f_{2}\right)=O\left(T\left(r, f_{1}\right)\right)+o\left(T\left(r, f_{1}\right)\right),
$$

where $r \rightarrow \infty$ outside of exceptional set $E$ with the property $\mathbb{P}$.
The following corollary of Theorem 5 is obtained by choosing $\mathcal{N}$ as the family of meromorphic functions of hyperorder strictly less than one with relatively few poles and by taking $L_{1}, \ldots L_{l+1}$ such that $L_{k}(f)=f\left(z+c_{k}\right), k=1, \ldots, l$, and $L_{l+1}(f)=f^{\prime}$.

Corollary 6. Let $M(z, f)$ be a differential-difference polynomial in $f$. If $f_{1}$ and $f_{2}$ are any two meromorphic solutions of (27) of hyperorder strictly less than one such that $N\left(r, f_{1}\right)=$ $S\left(r, f_{1}\right)$ and $N\left(r, f_{2}\right)=S\left(r, f_{2}\right)$, then

$$
T\left(r, f_{2}\right)=O\left(T\left(r, f_{1}\right)\right)+S\left(r, f_{1}\right) .
$$

Moreover, if $\alpha>1$, then, for some $r_{\alpha}>0$,

$$
T\left(r, f_{2}\right)=O\left(T\left(\alpha r, f_{1}\right)\right)
$$

for all $r \geq r_{\alpha}$. In addition, every meromorphic solution such that hyperorder $\sigma_{2}<1$ and $N(r, f)=S(r, f)$ satisfies $\rho(f)=$ $\rho(h)$.

Specialising to $M(z, f)-p(z) f^{3}=h(z)$, where $p(z)$ is a small meromorphic function, we obtain the following result on the existence of meromorphic solutions.

Theorem 7. Let $f$ be an transcendental meromorphic function of hyperorder $\sigma_{2}<1, M(z, f)$ a linear differential-difference polynomial of $f$ with small meromorphic coefficients, not vanishing identically, and $h$ a meromorphic function. Set $\lambda_{f}=$ $\max \{\lambda(f), \lambda(1 / f)\}$. If $f$ satisfies the nonlinear differentialdifference equation

$$
M(z, f)-p(z) f(z)^{3}=h(z),
$$

where $p(z)(\not \equiv 0)$ is a small function of $f$, then one of the following situations holds:

(a) Equation (31) has $f$ as its unique transcendental meromorphic solution such that $\lambda_{f}<\sigma_{f}$.

(b) Equation (31) has exactly three transcendental meromorphic solutions $f_{j}, j=1,2,3$, such that $\lambda_{f_{j}}<\sigma_{f_{j}}$ for $j=1,2,3$. Moreover $M\left(z, f_{j}\right) \equiv 0$, and $h(z)=$ $-p(z) f_{j}^{3}$ for all $j=1,2,3$.

If, in particular, we restrict the linear differential-difference polynomial $M(z, f)$ to be linear differential polynomial $L(z, f)$, then we get the following result which improves Theorem B.

Theorem 8. Let $f$ be a transcendental meromorphic function such that $N(r, f)=S(r, f)$. Moreover, let $k(\geq 1)$ be positive integer, let $p(z)(\neq \equiv 0)$ be a small function of $f$, and let $L(f)$ denote a linear differential polynomial in $f$ :

$$
L(f)=a_{0}(z) f+a_{1}(z) f^{\prime}+\cdots+a_{k}(z) f^{(k)},
$$

where $a_{j}(z)(j=0, \ldots, k)$ are small meromorphic functions such that not all $a_{j}$ are identically zero. Moreover, let $h(z)$ be a meromorphic function. If $f$ is a solution of the nonlinear differential equation

$$
L(f)-p(z) f(z)^{3}=h(z),
$$


then one of the following situations hold:

(a) Equation (33) has $f$ as its unique transcendental meromorphic solution such that $N(r, f)=S(r, f)$.

(b) Equation (33) has exactly three transcendental meromorphic solutions $f_{j}, j=1,2,3$, such that $N\left(r, f_{j}\right)=$ $S\left(r, f_{j}\right)$ for $j=1,2,3$. Moreover, $L\left(f_{j}\right) \equiv 0$, and $h(z)=-p(z) f_{j}^{3}$ for all $j=1,2,3$.

Following a similar method as in the proof of Theorems 7 and 8 , we can generalize the above two results to the case $P(z, f)=-p(z) f^{n}(z)$, where $n \geq 3$.

\section{Proof of Theorem 5}

By a repeated application of Lemma 1, it follows that, by composing finitely many good linear operators $\mathcal{N}$, we obtain another good linear operator for $\mathcal{N}$. Since $M(z, f)$ is a linear polynomial in $f$ and in the good linear operators $L_{j}(f)$, where $j \in J$, it follows that $M(z, f)$ can be written, without loss of generality, in the form

$$
M(z, f)=\sum_{j=1}^{p} a_{j}(z) \widetilde{L}_{j}(f(z)),
$$

where the coefficients $a_{j}$ are small meromorphic functions with respect to $f$ and $\widetilde{L}_{1}, \ldots, \widetilde{L}_{p}$ are good linear operators for $\mathcal{N}$ with exceptional set property $\mathbb{P}$.

Since $f_{1}$ and $f_{2}$ are solutions of (27), we have

$$
\begin{aligned}
& \frac{M\left(z, f_{1}\right)-M\left(z, f_{2}\right)}{f_{1}-f_{2}} \\
& =-\frac{P\left(z, f_{1}\right)-P\left(z, f_{2}\right)}{f_{1}-f_{2}} \\
& =-\frac{b_{n}\left(f_{1}^{n}-f_{2}^{n}\right)+\cdots+b_{2}\left(f_{1}^{2}-f_{2}^{2}\right)}{f_{1}-f_{2}}
\end{aligned}
$$

for some $n \geq 2$. Thus we have

$$
\begin{aligned}
\frac{M\left(z, f_{1}-f_{2}\right)}{f_{1}-f_{2}} & =\frac{M\left(z, f_{1}\right)-M\left(z, f_{2}\right)}{f_{1}-f_{2}} \\
& =Q\left(z, f_{1}, f_{2}\right),
\end{aligned}
$$

where $Q\left(z, f_{1}, f_{2}\right)$ is a polynomial in $f_{1}$ and $f_{2}$ with small meromorphic coefficients such that $\operatorname{deg}_{f_{1}} Q=\operatorname{deg}_{f_{2}} Q \geq$ 1. Now, since the coefficients $a_{j}$ in (34) are by assumption small with respect to both solutions $f_{1}$ and $f_{2}$, it follows that $T\left(r, a_{j}\right)=o\left(T\left(r, f_{1}\right)\right)$ and $T\left(r, a_{j}\right)=o\left(T\left(r, f_{2}\right)\right)$ for all $j \in\{1, \ldots, p\}$ as $r \rightarrow \infty$ outside of a set with exceptional set property $\mathbb{P}$. Hence,

$$
T\left(r, a_{j}\right)=o(T(r)), \quad j \in\{1, \ldots, p\},
$$

where

$$
T(r):=\max \left\{T\left(r, f_{1}\right), T\left(r, f_{2}\right)\right\}
$$

and $r \rightarrow \infty$ outside of a set with exceptional set property $\mathbb{P}$. Therefore, by using Lemma 1 repeatedly and by the definition of the good linear operator, we have

$$
\begin{aligned}
m & \left(r, Q\left(z, f_{1}, f_{2}\right)\right) \\
& =m\left(r, \frac{M\left(z, f_{1}-f_{2}\right)}{f_{1}-f_{2}}\right) \\
& \leq m\left(r, \frac{\sum_{j=1}^{p} a_{j}(z) \tilde{L}_{j}\left(f_{1}-f_{2}\right)}{f_{1}-f_{2}}\right) \\
& =o\left(T\left(r, f_{1}-f_{2}\right)\right)+o(T(r))=o(T(r)),
\end{aligned}
$$

where $r \rightarrow \infty$ outside of a set with exceptional set property $\mathbb{P}$.

Since by assumption (26) we have

$$
\max \left\{N\left(r, f_{1}\right), N\left(r, f_{2}\right)\right\}=o(T(r))
$$

as $r \rightarrow \infty$ outside of a set with exceptional set property $\mathbb{P}$, it follows that

$$
T\left(r, Q\left(z, f_{1}, f_{2}\right)\right)=o(T(r)),
$$

where again $r \rightarrow \infty$ outside of a set with exceptional set property $\mathbb{P}$. Therefore (36) becomes an algebraic equation for $f_{2}$ over the field

$$
\begin{aligned}
\mathscr{L} & :=\left\{h \text { meromorphic }: T(r, h)=O\left(T\left(r, f_{1}\right)\right)\right. \\
& +o(T(r))\} .
\end{aligned}
$$

Therefore, by a generalization of [16, Theorem 1] (see, e.g., [17, p. 34]), it follows that

$$
T\left(r, f_{2}\right)=O\left(T\left(r, f_{1}\right)\right)+o(T(r))
$$

as $r \rightarrow \infty$ outside of a set with exceptional set property $\mathbb{P}$. This asymptotic equation yields assertion (28).

\section{Proof of Corollary 6}

As a linear differential-difference polynomial, $M(z, f)$ may be written in the form

$$
M(z, f)=\sum_{i=0}^{k} \sum_{j=0}^{\ell} a_{i j}(z) f^{(j)}\left(z+c_{i}\right),
$$

where $k$ and $\ell$ are nonnegative integers, the coefficients $a_{i j}(z)$ are small meromorphic functions with respect to $f$, and $c_{0}, \ldots, c_{k}$ are complex constants. By defining $L_{i j}(f(z))=$ $f^{(j)}\left(z+c_{i}\right)$ and using Lemma 2, (44) becomes

$$
M(z, f)=\sum_{i=0}^{k} \sum_{j=0}^{\ell} a_{i j}(z) L_{i j}(f(z)),
$$

where $L_{i j}$ is a good linear operator in $\mathcal{N}_{1}$ for all $i$ and $j$ with the exceptional set property of "finite logarithmic measure." 
Hence Theorem 5 implies (29). By applying [17, Lemma 1.1.1] to remove the exceptional set, we get (30).

On the other hand, from (27), we have

$$
T(r, h) \leq T(r, M(z, f))+T(r, P(z, f))+O(1) .
$$

Since $a_{i j}$ are small functions for all $i=0, \ldots, k$ and $j=0, \ldots, \ell$ and $N(r, f)=S(r, f)$, by using Lemma 2 , we have

$$
\begin{aligned}
T(r, M(z, f))= & m(r, M(z, f))+N(r, M(z, f)) \\
\leq & m\left(r, \frac{M(z, f)}{f}\right)+m(r, f) \\
& +S(r, f) \\
\leq & \sum_{i=0}^{k} \sum_{j=0}^{\ell} m\left(r, a_{i j} \cdot \frac{L_{i j}(f)}{f}\right) \\
& +m(r, f)+S(r, f) \\
= & T(r, f)+S(r, f) .
\end{aligned}
$$

Further since $b_{j}$ are small functions for all $j=2, \ldots, n$, it follows from the Valiron-Mohon'ko theorem (see, e.g., [17, Theorem 2.2.5]) that

$$
T(r, P(z, f))=n T(r, f)+S(r, f) .
$$

Substituting (47) and (48) into (46) and applying [17, Lemma 1.1.1], we get that, for every $\varepsilon>0$ and $\alpha>1$, there exists $r_{\alpha}>0$ such that

$$
T(r, h) \leq(n+1+\varepsilon) T(\alpha r, f),
$$

provided $r \geq r_{\alpha}$. Then

$$
\begin{aligned}
\rho(h) & \leq \limsup _{r \rightarrow \infty} \frac{\log T(\alpha r, f)+O(1)}{\log r} \\
& =\limsup _{r \rightarrow \infty} \frac{\log T(\alpha r, f)}{\log \alpha r-\log \alpha}=\rho(f) .
\end{aligned}
$$

On the other hand, writing (27) in the form $P(z, f)=$ $-M(z, f)+h(z)$ and by making use of [17, Lemma 1.1.1], (47), and (48), we get that

$$
(n-1-\varepsilon) T(r, f) \leq T(\alpha r, h)
$$

when $r \geq r_{\alpha}$. Since $n \geq 2$ by the assumption, then from (51) we have

$$
\rho(f) \leq \limsup _{r \rightarrow \infty} \frac{\log T(\alpha r, h)}{\log (\alpha r)-\log \alpha}=\rho(h) .
$$

Thus $\rho(f)=\rho(h)$.

\section{Proof of Theorem 7}

Suppose that (31) has at least two distinct transcendental meromorphic solutions $f_{i}(i=1,2)$ such that $\lambda_{f_{i}}<\sigma_{f_{i}}$.
Then 0 and $\infty$ are two Borel exceptional values of $f_{i}$; thus $f_{i}$ is of regular growth. So we have $N\left(r, f_{i}\right)=S\left(r, f_{i}\right)$ and $N\left(r, 1 / f_{i}\right)=S\left(r, f_{i}\right)$. From (31), we have

$$
\begin{aligned}
\frac{M\left(z, f_{1}-f_{2}\right)}{f_{1}-f_{2}} & =\frac{M\left(z, f_{1}\right)-M\left(z, f_{2}\right)}{f_{1}-f_{2}} \\
& =p(z) \frac{f_{1}^{3}-f_{2}^{3}}{f_{1}-f_{2}}=p(z) F(z),
\end{aligned}
$$

where $F(z)=\left(f_{1}-\varepsilon_{1} f_{2}\right)\left(f_{1}-\varepsilon_{2} f_{2}\right)$. Here $\varepsilon_{j} \neq 1$ are the distinct third roots of unity. Also, recalling the definition of $T(r)$ from (38), we have that $T(r, p)=o(T(r))$ as $r \rightarrow \infty$ outside of an exceptional set of finite logarithmic measure. Thus by Lemmas 1 and 2 it follows that

$$
\begin{aligned}
m(r, F) & =m\left(r, \frac{1}{p} \cdot \frac{M\left(z, f_{1}-f_{2}\right)}{f_{1}-f_{2}}\right) \\
& =S\left(r, f_{1}-f_{2}\right)+o(T(r))=o(T(r)),
\end{aligned}
$$

where $r \rightarrow \infty$ outside of an exceptional set of finite logarithmic measure. Moreover,

$$
N(r, F)=o(T(r)),
$$

and thus

$$
T(r, F)=o(T(r))
$$

again as $r \rightarrow \infty$ outside of an exceptional set of finite logarithmic measure. Assume first that $F \equiv 0$. Then, by the definition of $F$, it follows that $f_{2}=\varepsilon_{j} f_{1}$ for some $j=1,2$. Substituting this into (53), we have

$$
M\left(z, f_{1}-\varepsilon_{j} f_{1}\right)=\left(1-\varepsilon_{j}\right) M\left(z, f_{1}\right)=0,
$$

and so $M\left(z, f_{1}\right)=0$. Thus from (31) we have $h(z)=$ $-p(z) f_{1}^{3}(z)$ as asserted. Moreover, $M\left(z, \varepsilon_{j} f_{1}\right)=0$ and thus $h(z)=-p(z)\left(\varepsilon_{j} f_{1}(z)\right)^{3}$ for both $j=1$ and $j=2$. Therefore, $f_{1}, \varepsilon_{1} f_{1}$, and $\varepsilon_{2} f_{1}$ are the solutions of (31), and so we have completed the proof of part (b).

Assume now that $F \neq \equiv$. Then

$$
\begin{aligned}
F(z) & =f_{1}^{2}\left(1-\varepsilon_{1} \frac{f_{2}}{f_{1}}\right)\left(1-\varepsilon_{2} \frac{f_{2}}{f_{1}}\right) \\
& =\varepsilon_{1} \varepsilon_{2} f_{1}^{2}\left(\varepsilon_{1}^{2}-\frac{f_{2}}{f_{1}}\right)\left(\varepsilon_{2}^{2}-\frac{f_{2}}{f_{1}}\right) .
\end{aligned}
$$

By (58), we have that

$$
\frac{F(z)}{f_{1}^{2}}=Q\left(\frac{f_{2}}{f_{1}}\right),
$$

where $Q$ is a polynomial in $f_{2} / f_{1}$ of degree 2 with constant coefficients. Since $T(r, F)=o(T(r))$, we have

$$
\begin{aligned}
2 T\left(r, \frac{f_{2}}{f_{1}}\right)+O(1) & =T\left(r, \frac{F}{f_{1}^{2}}\right) \\
& =2 T\left(r, f_{1}\right)+o(T(r))
\end{aligned}
$$


as $r \rightarrow \infty$ outside of an exceptional set of finite logarithmic measure. Similarly,

$$
\begin{aligned}
2 T\left(r, \frac{f_{2}}{f_{1}}\right)+O(1) & =2 T\left(r, \frac{f_{1}}{f_{2}}\right)+O(1) \\
& =T\left(r, \frac{F}{f_{2}^{2}}\right) \\
& =2 T\left(r, f_{2}\right)+o(T(r)),
\end{aligned}
$$

where, as above, $r \rightarrow \infty$ outside of an exceptional set of finite logarithmic measure. Combining (60) and (61), we obtain

$$
T\left(r, f_{1}\right)=T\left(r, f_{2}\right)+o(T(r)),
$$

where the exceptional set is as above. Hence $S\left(r, f_{1}\right)=$ $S\left(r, f_{2}\right)$, and so by $(60)$ we have

$$
T\left(r, \frac{f_{2}}{f_{1}}\right)=T\left(r, f_{1}\right)+S\left(r, f_{1}\right) .
$$

Thus the error terms $o(T(r))$ and $S\left(r,\left(f_{2} / f_{1}\right)\right)$ are asymptotically equivalent. Assuming that $\left(f_{2} / f_{1}\right)\left(z_{0}\right)=\varepsilon_{j}^{2}$ for some $j=1,2$, then from (58) we have $F\left(z_{0}\right)=0$, or $f_{1}\left(z_{0}\right)=\infty$. So we have

$$
\bar{N}\left(r, \frac{1}{\left(f_{2} / f_{1}\right)-\varepsilon_{j}^{2}}\right)=S\left(r, \frac{f_{2}}{f_{1}}\right), \quad j=1,2 .
$$

Since

$$
\begin{aligned}
\bar{N}\left(r, \frac{1}{\left(f_{2} / f_{1}\right)}\right) & =\bar{N}\left(r, \frac{f_{1}}{f_{2}}\right) \\
& \leq \bar{N}\left(r, f_{1}\right)+\bar{N}\left(r, \frac{1}{f_{2}}\right) \\
& =S\left(r, \frac{f_{2}}{f_{1}}\right),
\end{aligned}
$$

it follows by the second main theorem of Nevanlinna theory that

$$
\begin{aligned}
T\left(r, \frac{f_{2}}{f_{1}}\right) \leq & \bar{N}\left(r, \frac{1}{f_{2} / f_{1}}\right)+\bar{N}\left(r, \frac{1}{\left(f_{2} / f_{1}\right)-\varepsilon_{1}^{2}}\right) \\
& +\bar{N}\left(r, \frac{1}{\left(f_{2} / f_{1}\right)-\varepsilon_{2}^{2}}\right)=S\left(r, \frac{f_{2}}{f_{1}}\right) .
\end{aligned}
$$

This is a contradiction. Thus (31) may have only one transcendental meromorphic solution such that $\lambda_{f}<\sigma_{f}$, as asserted in part (a).

\section{Proof of Theorem 8}

Suppose that (33) has at least two distinct transcendental meromorphic solutions $f_{i}(i=1,2)$ such that $N\left(r, f_{i}\right)=$ $S\left(r, f_{i}\right)$. Then, similarly as in the proof of Theorem 7 ,

$$
\begin{aligned}
& \frac{\sum_{i=0}^{k} a_{i}(z)\left(f_{1}-f_{2}\right)^{(i)}}{f_{1}-f_{2}} \\
& \quad=p(z) \frac{f_{1}^{3}-f_{2}^{3}}{f_{1}-f_{2}}=p(z) F(z),
\end{aligned}
$$

where $F(z)=\left(f_{1}-\varepsilon_{1} f_{2}\right)\left(f_{1}-\varepsilon_{2} f_{2}\right), T\left(r, a_{i}\right)=o(T(r))$, and $T(r, p)=o(T(r))$ with the error term $o(T(r))$ being defined as in (38) and $r \rightarrow \infty$ outside of an exceptional set of finite linear measure. Here $\varepsilon_{j} \neq 1$ are the distinct third roots of unity. Also, we have

$$
\begin{aligned}
m(r, F) & =m\left(r, \frac{1}{p} \cdot \frac{\sum_{i=0}^{k} a_{i}(z)\left(f_{1}-f_{2}\right)^{(i)}}{f_{1}-f_{2}}\right) \\
& =S\left(r, f_{1}-f_{2}\right)+o(T(r))=o(T(r)),
\end{aligned}
$$

where $r \rightarrow \infty$ outside of an exceptional set of finite linear measure, and

$$
N(r, F)=o(T(r))
$$

by assumption. The case $F=0$ leads to the assertion of part (b) exactly as in the proof of Theorem 7, and so we may assume that $F \neq 0$. Again, by repeating the reasoning in the proof of Theorem 7, we have that $T(r, F)=S\left(r, f_{2} / f_{1}\right)$ and

$$
\bar{N}\left(r, \frac{1}{\left(f_{2} / f_{1}\right)-\varepsilon_{j}^{2}}\right)=S\left(r, \frac{f_{2}}{f_{1}}\right), \quad j=1,2 .
$$

Assume now that there exists $z_{0} \in \mathbb{C}$ such that $\left(f_{2} / f_{1}\right)\left(z_{0}\right)=$ 1. Then $\left(f_{1}-f_{2}\right)\left(z_{0}\right)=0$. From (67), we have

$$
\begin{aligned}
\bar{N}\left(\frac{1}{f_{1}-f_{2}}\right) \leq & \sum_{i=0}^{k}\left(N\left(r, a_{i}(z)\right)+N\left(r, \frac{1}{a_{i}(z)}\right)\right) \\
& +N(r, p(z))+N(r, F(z)) \\
& +S\left(r, \frac{f_{2}}{f_{1}}\right)=S\left(r, \frac{f_{2}}{f_{1}}\right) .
\end{aligned}
$$

Thus we get

$$
\bar{N}\left(r, \frac{1}{\left(f_{2} / f_{1}\right)-1}\right)=S\left(r, \frac{f_{2}}{f_{1}}\right) .
$$

So, by (70), (72), and the second main theorem of Nevanlinna theory, we get

$$
\begin{aligned}
T\left(r, \frac{f_{2}}{f_{1}}\right) \leq & \bar{N}\left(r, \frac{1}{\left(f_{2} / f_{1}\right)-1}\right) \\
& +\bar{N}\left(r, \frac{1}{\left(f_{2} / f_{1}\right)-\varepsilon_{1}^{2}}\right) \\
& +\bar{N}\left(r, \frac{1}{\left(f_{2} / f_{1}\right)-\varepsilon_{2}^{2}}\right)=S\left(r, \frac{f_{2}}{f_{1}}\right) .
\end{aligned}
$$

This is a contradiction. Therefore (33) may have only one transcendental meromorphic solution such that $N(r, f)=$ $S(r, f)$, as asserted in part (a).

\section{Conflict of Interests}

The authors declare that there is no conflict of interests regarding the publication of this paper. 


\section{Acknowledgments}

This work was supported by the NNSF of China (no. 11171013 and no. 11371225) and the Academy of Finland Grant no. 268009.

\section{References}

[1] J. Clunie, "On integral and meromorphic functions," Journal of the London Mathematical Society, vol. 37, pp. 17-27, 1962.

[2] A. Z. Mohon'ko and V. D. Mohon'ko, "Estimates of the Nevanlinna characteristics of certain classes of meromorphic functions, and their applications to differential equations," Sibirskii Matematicheskii Zhurnal, vol. 15, pp. 1305-1322, 1974 (Russian).

[3] R. G. Halburd and R. J. Korhonen, "Difference analogue of the lemma on the logarithmic derivative with applications to difference equations," Journal of Mathematical Analysis and Applications, vol. 314, no. 2, pp. 477-487, 2006.

[4] R. G. Halburd and R. J. Korhonen, "Nevanlinna theory for the difference operator," Annales Academice Scientiarum Fennica. Mathematica, vol. 31, no. 2, pp. 463-478, 2006.

[5] Y.-M. Chiang and S.-J. Feng, "On the Nevanlinna characteristic of $f(z+\eta)$ and difference equations in the complex plane," The Ramanujan Journal, vol. 16, no. 1, pp. 105-129, 2008.

[6] Y.-M. Chiang and S.-J. Feng, "On the growth of logarithmic differences, difference quotients and logarithmic derivatives of meromorphic functions," Transactions of the American Mathematical Society, vol. 361, no. 7, pp. 3767-3791, 2009.

[7] D. C. Barnett, R. G. Halburd, W. Morgan, and R. J. Korhonen, "Nevanlinna theory for the $q$-difference operator and meromorphic solutions of $q$-difference equations," Proceedings of the Royal Society of Edinburgh. Section A. Mathematics, vol. 137, no. 3, pp. 457-474, 2007.

[8] R. Korhonen, "An extension of Picard's theorem for meromorphic functions of small hyper-order," Journal of Mathematical Analysis and Applications, vol. 357, no. 1, pp. 244-253, 2009.

[9] R. Halburd and R. Korhonen, "Value distribution and linear operators," Proceedings of the Edinburgh Mathematical Society, vol. 57, no. 2, pp. 493-504, 2014.

[10] R. G. Halburd, R. Korhonen, and K. Tohge, "Holomorphic curves with shift-invariant hyperplane preimages," Transactions of the American Mathematical Society, vol. 366, no. 8, pp. 42674298, 2014.

[11] J. Heittokangas, R. Korhonen, and I. Laine, "On meromorphic solutions of certain nonlinear differential equations," Bulletin of the Australian Mathematical Society, vol. 66, no. 2, pp. 331-343, 2002.

[12] C.-C. Yang and I. Laine, "On analogies between nonlinear difference and differential equations," Proceedings of the Japan Academy, Series A, Mathematical Sciences, vol. 86, no. 1, pp. 10$14,2010$.

[13] Z. T. Wen, J. Heittokangas, and I. Laine, "Exponential polynomials as solutions of certain nonlinear difference equations," Acta Mathematica Sinica, vol. 28, no. 7, pp. 1295-1306, 2012.

[14] S. Wang and S. Li, "On entire solutions of nonlinear differencedifferential equations," Bulletin of the Korean Mathematical Society, vol. 50, no. 5, pp. 1471-1479, 2013.

[15] X. Qi and L. Yang, "Properties of meromorphic solutions to certain differential-difference equations," Electronic Journal of Differential Equations, No. 135, 9 pages, 2013.
[16] S. Hellerstein and L. A. Rubel, "Subfields that are algebraically closed in the field of all meromorphic functions," Journal d'Analyse Mathématique, vol. 12, pp. 105-111, 1964.

[17] I. Laine, Nevanlinna Theory and Complex Differential Equations, vol. 15 of De Gruyter Studies in Mathematics, Walter de Gruyter, Berlin, Germany, 1993. 


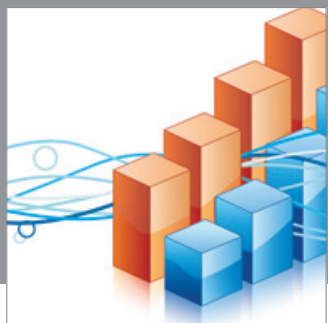

Advances in

Operations Research

mansans

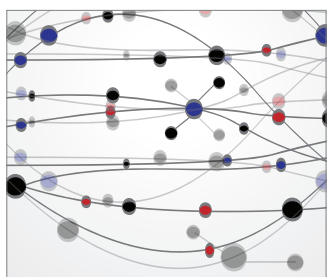

The Scientific World Journal
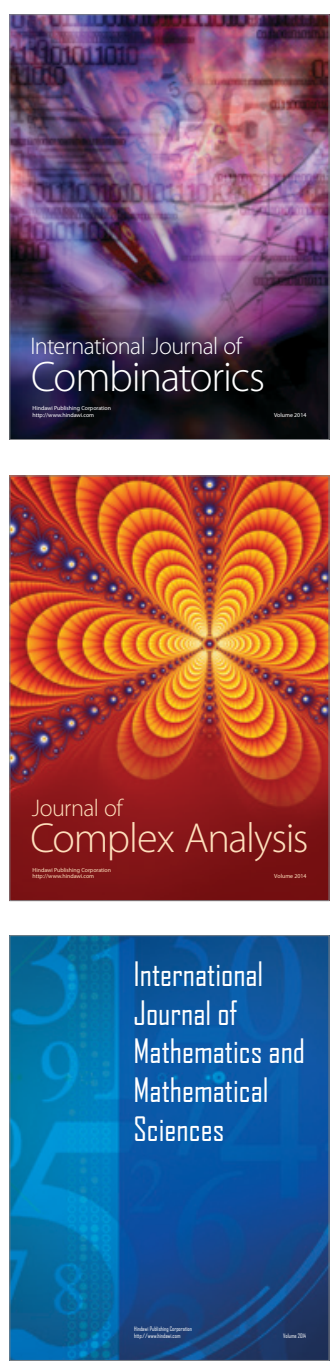
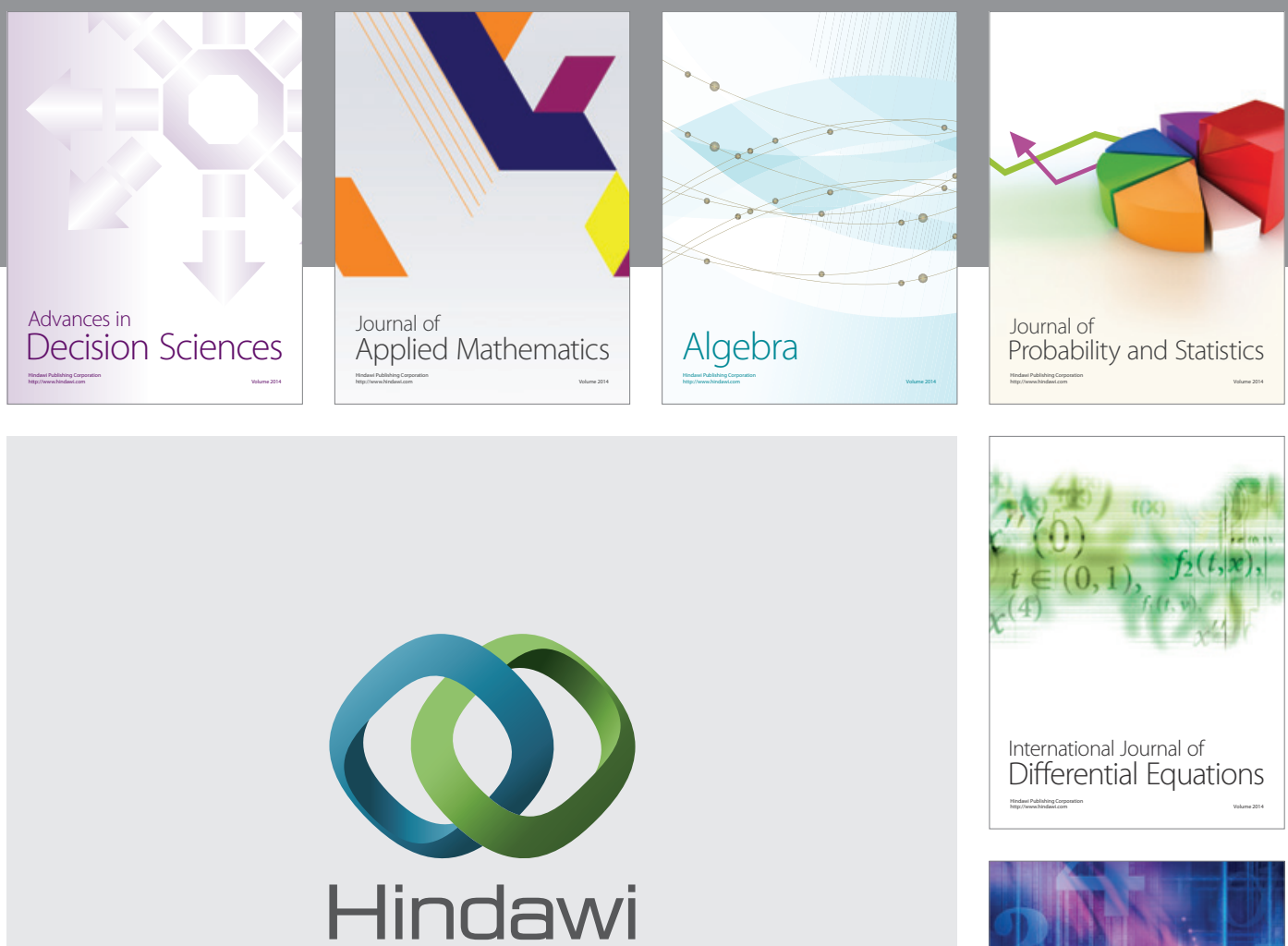

Submit your manuscripts at http://www.hindawi.com
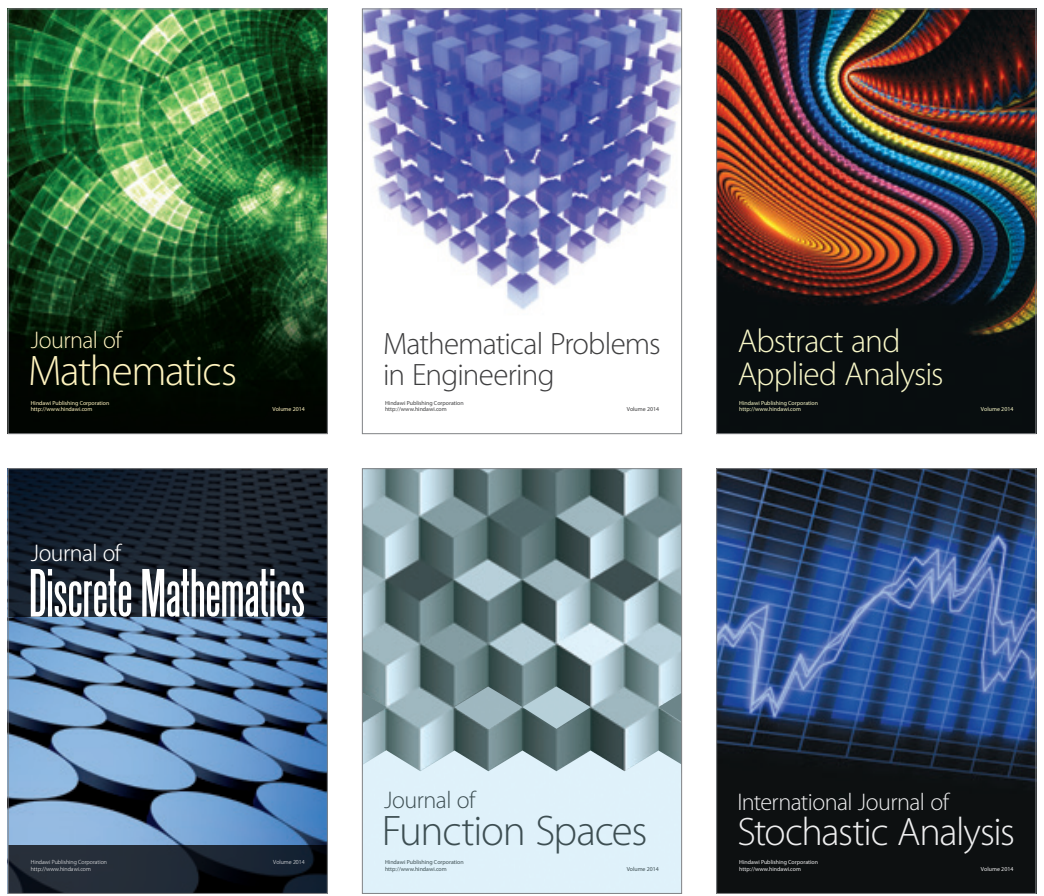

Journal of

Function Spaces

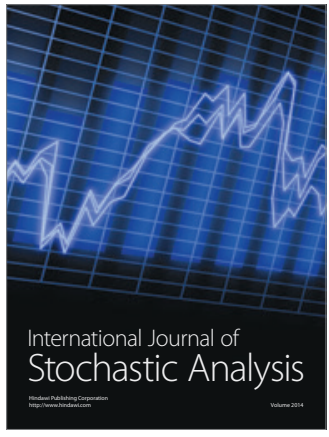

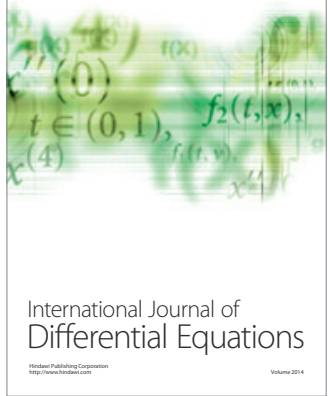
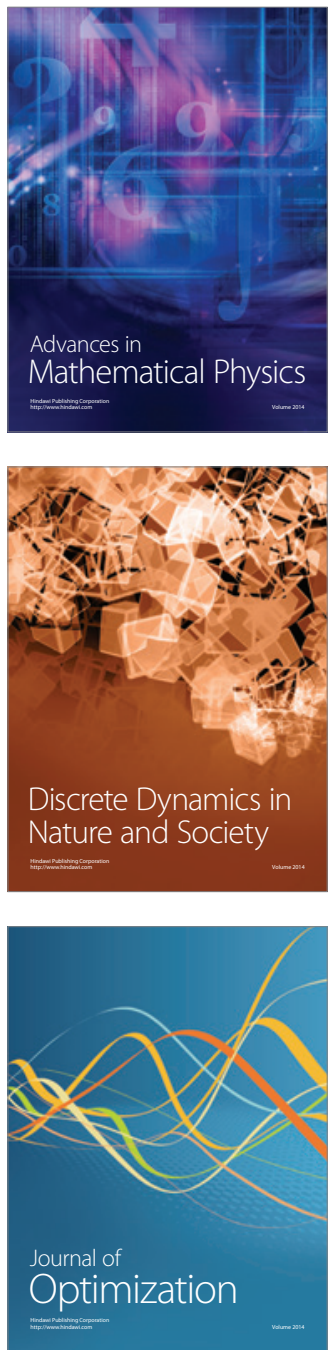